5. Пат. 2251081 Российская Федерация, МПК7 G 01 F 1/32, 15/12. Счетчик-расходомер / Кобелев Н.С., Гнездилова О.А., Захаров И.С.; заявитель и патентообладатель КурскГТУ. - № 2003112072/28; заявл. 24.04.2003.; опубл. 27.04.2005, Бюл. № 12 (II ч.).

Гнездилова Ольга Александровна, канд. техн. наук, доцент кафедры «Теплогазоснабжение и вентиляцуия», Национальный исследовательский Московский государственный строительный университет. Россия.

129337, г. Москва, Ярославское шоссе, 26.

Тел.: (495) 781-80-07.

Ключевые слова: расход газа; вихревой счетчик-расходомер; ЭВМ-контроль; система газоснабжения.

\title{
DEVELOPMENT OF A COMPUTER PROGRAM FOR AUTOMATED CONTROL OF METERING OF SMALL GAS FLOWS
}

Gnezdilova Olga Akeksandrovna, Candidate of Technical Sciences, Associate Professor of the chair "Heat and Gas Supply and Ventilation", National Research Moscow State University of Civil Engineering. Russia.

Keywords: gas consumption; swirl counter flow meter; computer control; gas supply system.

The problem of reliability of energy systems, which include the gas supply system, are considered. Special attention is paid to the development of the description of the computer-control program for metering of low gas consumption "Energy resources control: KER 1-gas". The description of the tasks is given taking into account the accepted conditions of the constructive, technological and production-organizational plan.

\section{ТРАЕКТОРНЫЙ АНАЛИЗ ЗАРЯЖЕННОЙ ЧАСТИЦЫ}

\begin{abstract}
КУЗЬМИН Константин Анатольевич, Московский государственный университет технологий и управления имени К.Г. Разумовского (Первый казачий университет)
\end{abstract}

МОРОЗОВ Сергей Михайлович, Московский государственный университет технологий и управления имени К.Г. Разумовского (Первый казачий университет)

РОГОЖИНА Анна Владимировна, Национальный исследовательский Московский государственный строительный университет

Проводится анализ поведения заряженной частицы в неоднородном электрическом поле точечного источника. Построена математическая модель и приведено решение системы дифференциальных уравнений в частных производных.

Введение. Проблема анализа движения частицы имеет фундаментальное значение. В процессах преобразования потока заряженных частиц во многих физических устройствах она может рассматриваться как задача о движении частицы в неоднородном стационарном и нестационарном потенциальном поле (электрическое, термоэлектрическое, гравитационное и т.д.) [4-6]. Неоднородное потенциальное поле может создаваться, например, точечным источником и другими различными физическими структурами. Одной из таких физических систем является плоская структура, состоящая из двух расположенных под углом друг другу электродов.

Такая система с неоднородным элект- рическим полем может быть использована для разработки устройств измерения и функционального преобразования электрических сигналов как в твердотельных, так и в вакуумных структурах [2]. Наиболее ярким примером неоднородного поля в полупроводниковых устройствах является граница металл-полупроводник (MS), создаваемого неподвижным пространственным зарядом. Взаимодействие частицы с ним является одним из главных факторов, определяющих характеристику преобразования рассматриваемого устройства. Поэтому знание физических процессов на границе раздела поверхности металл-полупроводник (барьер Шоттки) имеет фундаментальное значение для понимания 
принципа работы многих полупроводниковых приборов и определяет широкий интерес к теоретическим и экспериментальным работам в этом направлении [1]. К ним можно отнести исследования закономерностей возникновения нелинейной вольтамперной характеристики в микро- и макроскопических образцах полупроводниковых устройств.

Нелинейность происходит из-за сложного механизма физических процессов, происходящих на границах сплошных сред при большом разнообразии потенциальных барьеров [2,3]. Кроме того, решение этой задачи позволило бы, вероятно, получить обобщенную картину поведения частиц в ускоряющих и тормозящих термоэлектрических потенциальных полях и исследовать общие закономерности преобразования электронного потока в физических устройствах. Наиболее подробно процессы транспорта носителей зарядов в ней рассмотрены в [3], но теоретический анализ потенциального барьера полупроводниковых структур - нетривиальная задача даже для простого линейного полевого профиля. Вследствие фундаментальной ценности понимания физических процессов энергетического преобразования частиц теоретические и экспериментальные работы в этом направлении носят фундаментальный характер и являются актуальными в настоящее время. Процессы преобразования кинетической энергии потока заряженных частиц во многих физических устройствах с неоднородными полями не всегда удается аналитически описать известными закономерностями движения частицы в однородном потенциальном поле. Различные подходы к теоретическому анализу, от простых моделей Левинсона до решений уравнений Лапласа или Пуассона и квантомеханического анализа процесса, по разным причинам не позволяют полностью исследовать физическую картину процесса преобразования. Аналитическое решение такой проблемы в большинстве случаев невозможно, и только численное решение позволяет судить о физическом процессе, что часто затрудняет понимание сути рассматриваемых явлений. Кроме того, численное решение не позволяет всесторонне (только в заданной области потенциального поля) анализировать движение частицы, а также может содержать информацию, вызванную вычислительными ошибками. В стационарных потенциальных полях (электрические, тепловые и гравитационные) задача о движении частиц, как правило, сводится к вычислению параметров поля (обычно расчета потенциала, удовлетворяющего уравнению Лапласа) и решению самого уравнения движения частиц в поле с этим потенциалом. Аналитическое решение уравнения движения в системе центральных сил получено в ограниченном числе вариантов распределения потенциала. Трудности аналитического описания возрастают при движении заряженной частицы в электрическом поле при влиянии величины его заряда на распределение этого поля.

Методика исследования. Задачи решаются методом запаздывающих потенциалов, что представляет собой итерационный процесс, что в свою очередь затрудняет понимание физического процесса. Поэтому аналитическое решение классических уравнений движения частицы в потенциальном неоднородном поле остается важной научной проблемой. Следует отметить, что знания закономерностей движения частицы в потенциальном неоднородном поле будут полезны и для практики создания физических устройств.

Поле точечного источника рассматривается как симметричное поле с распределенным потенциалом (см. рисунок, кривые 1), в котором его потенциал описывается равнобокими гиперболами, асимптотами которых являются оси $X$ и $Y$, а его параметр равен половине значения коэф-

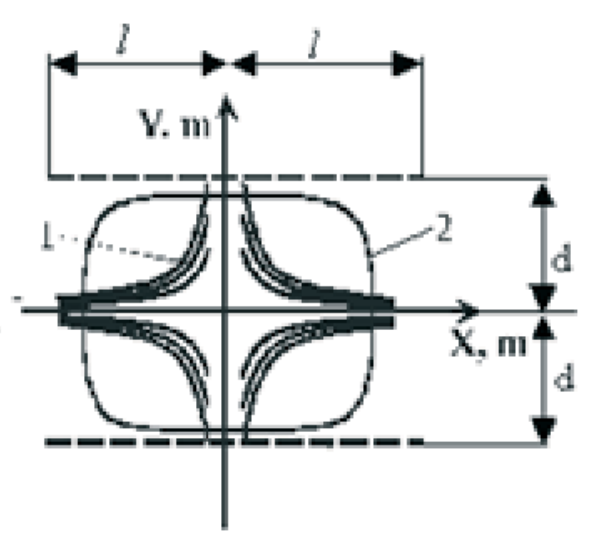

Поле точечного источника 
фициента распределения потенциала.

Уравнение поля точечного заряда (источника):

$U(x, y)= \pm \frac{\left(U_{\max }-U_{\min }\right)}{l^{2}} x y \mp U_{0} \cdot= \pm q \frac{\left(l-r_{0}\right)}{l^{3} \cdot r_{0}} x y \mp U_{0}$,

где, $k$ - коэффициент распределения потенциала, $k=\left(U_{0}-U_{\min }\right) / l^{2}, U_{\min }-$ минимальное значение потенциала (значение потенциала на длине 1 в исследуемой области), $U_{\min }=q / l ; U_{0}-$ максимальное значение потенциала точечного источника в его окрестности на радиусе $r_{0}, U_{0}=q / r_{0}$; $q$ - величина заряда точечного источника; $r_{0}$ - величина радиуса окрестности точечного источника, (на котором определяется величина потенциала $\left.U_{0}\right)$.

Уравнение движения частицы (например, электрон) в поле точечного источника имеет следующий вид:

$$
\left\{\begin{array}{l}
\ddot{x}=\mp e m^{-1} E_{x}=\mp \alpha_{s} y, \\
\ddot{y}=\mp e m^{-1} E_{y}=\mp \alpha_{s} x,
\end{array}\right.
$$

где $\alpha$-параметр уравнения (положителен во втором и четвертом квадрантах и отрицателен в первом и третьем квадрантах)

Решение уравнения движения имеет следующий вид:

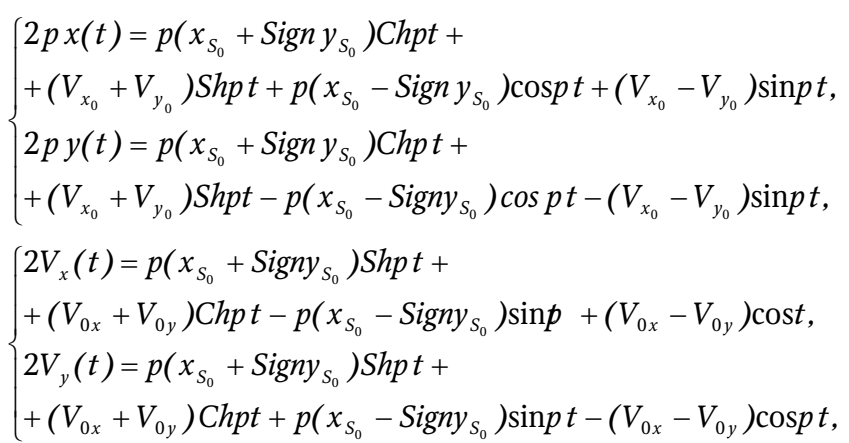

где $x_{S_{0}}, y_{S_{0}}, V_{x_{0}}, V_{y_{0}}$ - соответственно начальные значения координат и скорости, влетающей частицы в неоднородное поле точечного

источника Sign $y=\left\{\begin{array}{r}1 \text {, at } y>0, \\ -1 \text {, at } y<0 .\end{array}\right.$

Это все относится к слаботочным устройствам, т.е. там, где частица не влияет на поле точечного источника. В случае сильноточных устройств эти уравнения не работают.

Такие задачи, как правило, решаются с помощью запаздывающих потенциалов, что вызывает определенные трудности.

Результатъи исследований. В данной работе предлагается аналитически описать движение частицы с учетом ее влияния на потенциальное поле точечного источника.

Поле двух неподвижных точечных зарядов:

$$
U(x, y)= \pm k_{1} x y \mp U_{01} \cdot \pm k_{2} x y \mp U_{02} .
$$

При движении первого заряда суммарное поле

$U(x, y, t)= \pm k_{1}(x+\Delta x)(y+\Delta y) \mp U_{01} \cdot \pm k_{2} x y \mp U_{02}$.

Или

$U(x, y, t)= \pm k_{1}(x+\dot{x} \cdot t)(y+\dot{y} \cdot t) \mp U_{01} \cdot \pm k_{2} x y \mp U_{02} ;$

$$
\begin{aligned}
& U(x, y, t)= \pm k_{1} x y \pm k_{1} y \dot{x} \cdot t \pm k_{1} x \dot{y} t \pm \\
& \pm k_{1} \dot{x} t \dot{y} t \mp U_{01} \cdot \pm k_{2} x y \mp U_{02}
\end{aligned}
$$

$E=\operatorname{grad} U(x, y, t)= \pm k_{1}(x+\dot{x} t)(y+\dot{y} t) \pm$; $\mp U_{01} \cdot \pm k_{2} x y \mp U_{02}$

$$
\begin{gathered}
E(x, t)= \pm k_{1} \cdot y \pm k_{1} \dot{y} t \pm k_{2} y= \pm\left(k_{1}+ \pm k_{2}\right) y \pm k_{1} \dot{y} t ; \\
E(y, t)= \pm k_{1} x \pm k_{1} \dot{x} t \pm k_{2} x= \pm\left(k_{1}+ \pm k_{2}\right) x \pm k_{1} \dot{x} t
\end{gathered}
$$

Уравнение движения первой частицы в поле точечного источника имеет следующий вид:

$$
\left\{\begin{array}{l}
\ddot{x}=\mp e m^{-1} E(x, t)=\mp \alpha_{S}\left(\left(k_{1}+k_{2}\right) y \pm k_{1} y t\right), \\
\ddot{y}=\mp e m^{-1} E(y, t)= \pm \alpha_{S}\left(\left(k_{1}+k_{2}\right) x \pm k_{1} \dot{x} t\right) .
\end{array}\right.
$$

Решим это уравнение:

$$
\begin{aligned}
& \ddot{y}-\ddot{x}=\left(\mp \alpha_{S}\left(\left(k_{1}+k_{2}\right) \cdot x \pm k_{1} \dot{x} t\right)\right)- \\
& -\left(\mp \alpha_{S}\left(\left(k_{1}+k_{2}\right) y \pm k_{1} \dot{y} \cdot t\right)\right)= \\
& =\mp \alpha_{S}\left(\left(k_{1}+k_{2}\right)(x-y) \pm k_{1}(\dot{x}-\dot{y}) t\right)
\end{aligned}
$$

Обозначим $\mp \alpha_{S} *\left(\left(k_{1}+k_{2}\right)=-\mathrm{A}, \pm k_{1}=-\mathrm{B}\right.$. Получим уравнение:

$$
\ddot{y}-\ddot{x}=\mathrm{A}(y-x)+B(\dot{y}-\dot{x}) t .
$$

Введем новую переменную $z=y-x$. Полагая независимость $x$ и $y$, получим:

$$
\begin{gathered}
\ddot{z}=\mathrm{A} z+B \dot{z} t ; \\
\ddot{z}-B \dot{z} t-\mathrm{A} z=0 .
\end{gathered}
$$

Рассмотрим несколько частных случаев. 1. $A=0$. Получим: 


$$
\begin{aligned}
& \dot{z}=p(t) ; \ddot{z}=\dot{p} ; \\
& \dot{p}-B p t=0 ; \frac{\dot{p}}{p}=B p ; \\
& \frac{d p}{p}=B d t ; \ln p=B t+C ; \\
& p=C_{1} e^{B t} . \\
& \frac{d z}{d t}=C_{1} e^{B t} ; \\
& z=C_{1} \int e^{B t} d t+C_{2} ; \\
& z=\frac{C_{1}}{B} e^{B t}+C_{2} ; \\
& y-x=\frac{C_{1}}{B} e^{B t}+C_{2} . \\
& \ddot{z}-B \dot{z} t=0
\end{aligned}
$$

2. $B=0$.

$$
\begin{aligned}
& \ddot{z}-\mathrm{A} z=0 ; \dot{z}=p(z) \\
& \ddot{z}=\dot{p} p ; \dot{p} p-A z=0 ; \\
& \dot{p} p=A z ; p d p=A z d z ; \\
& \frac{p^{2}}{2}=A \frac{z^{2}}{2}+C_{11} ; p= \pm \sqrt{A z^{2}+C_{1}^{2}} ; \\
& \frac{d z}{d t}= \pm \sqrt{A z^{2}+C_{1}^{2}} ; \frac{d z}{ \pm \sqrt{A z^{2}+C_{1}^{2}}}=d t ; \\
& \ln \left(z+\sqrt{A z^{2}+C_{1}^{2}}\right)=t+C_{22} ; \\
& z+\sqrt{A z^{2}+C_{1}^{2}}=C_{2} e^{t} ; \\
& y-x+\sqrt{A z^{2}+C_{1}^{2}}=C_{2} e^{t} . \\
& 3 . A=B=M . \\
& \ddot{z}-M(\dot{z} t+z)=0 ; \ddot{z}=M(\dot{z} t+z) ; \\
& \ddot{z}=(M z t)^{\prime} ; \dot{z}=M z t+C_{1} ; \dot{z}-M z t=C_{1} .
\end{aligned}
$$

Решаем однородное уравнение:

$$
\begin{aligned}
& \dot{z}-M z t=0 ; \frac{\dot{z}}{z}=M z t ; \\
& \frac{d z}{z}=M t d t ; \ln z=\int M t d t+C_{11} ; \\
& h z=M \frac{t^{2}}{2}+C_{1} ; z=C(t) e^{M \frac{t^{2}}{2}} ; \\
& \dot{z}=\dot{C} e^{M \frac{t^{2}}{2}}+C M t e^{M \frac{t^{2}}{2}} .
\end{aligned}
$$

Решаем неоднородное уравнение:

$$
\begin{aligned}
& \dot{C} e^{M \frac{t^{2}}{2}}+C M t e^{M \frac{t}{2}}-C M t e^{M \frac{t^{2}}{2}}=C_{1} ; \\
& \dot{C} e^{M \frac{t^{2}}{2}}=C_{1} ; C(t)=C_{1} \int e^{-M \frac{t^{2}}{2}} d t+C_{2} .
\end{aligned}
$$

Таким образом, получим

$$
\begin{aligned}
& z=\left(C_{1} \int e^{-M \frac{t^{2}}{2}} d t+C_{2}\right) e^{M \frac{t^{2}}{2}} \\
& y-x=\left(C_{1} \int e^{-M \frac{t^{2}}{2}} d t+C_{2}\right) e^{M \frac{t^{2}}{2}}
\end{aligned}
$$

При решении конкретных прикладных задач можно воспользоваться таблицами для определенного интеграла.

Заключение. В работе аналитически описано движение частицы с учетом ее влияние на потенциальное поле точечного источника. Представлена система дифференциальных уравнений второго порядка и приведено решение системы для различных частных случаев.

\section{СПИСОК ЛИТЕРАТУРЫ}

1. Федулов В.И., Захидов Р.А., Анарбаев А. Частица в неоднородном потенциальном поле устройств фотовольтаики // Гелеотехника. - 2009. - № 3. С. 28-36.

2. Федулов В.И., Морозов С.М. Потери кинетической энергии частицы в неоднородном потенциальном поле // Современное телевидение и радиоэлектроника: 19-я Междунар. науч.-техн. конф. -М., 2011. - С. 299-301.

3. Федулов В.И., Морозов С.М. О нелинейных характеристиках структур с неоднородным потенциальным полем // Современное телевидение и радиоэлектроника: 19-я Междунар. науч.техн. конф. -М., 2011. - С. 301-304.

4. Fedulov V.I. ARW977788 Emerging Applications of Vacuum-Arc-Produced Plasma, Ion and Electron Beams, ed. by E.M. Oks and I.G. Brown (Kluwer Academic Publishers, Dordrecht, the Netherlands), 2003; 213-225.

5. Kroemer Herbert. Electrical and Computer Engineering Department, University of California, Santa Barbara, 2001.

6. Landau L., Lifchic E. The theory of a field. Moscow, 1998, Vol. 2, p. 78-79.

Кузьмин Константин Анатольевич, канд. пед. наук, доцент кафедры «Естественно-научные технические дисциплины и информационные технологии», Московский государственный университет технологий и управления имени К.Г. Разумовского (Первый казачий университет). Россия.

Морозов Сергей Михайлович, канд. техн. наук, доцент, зав. кафедрой «Естественно-научные технические дисииплины и информационные технологии», Московский государственный университет технологий и управления имени К.Г. Разумовского (Первый казачий университет). Россия.

215100, Смоленская обл., г. Вязьма, ул. Ленина, 77. 
Тел.: (48131) 6-18-66.

Рогожина Анна Владимировна, преподаватель кафедры «Металлические и деревянные конструкции», Национальный исследовательский Московский государственный строительный университет. Россия.
129337, г. Москва, Ярославское шоссе, 26.

Тел.: (495) 781-80-07.

Ключевые слова: заряженная частииа; неоднородное электрическое поле; точечный источник; математическая модель; система дифференииильных уравнений; поле точечного источника.

\title{
CHARGED SIDE TRAJECTORY ANALYSIS
}

Kuzmin Konstantin Anatolyevich, Candidate of Pedagogical Sciences, Associate Professor of the chair "Science and Technical Disciplines and Information Technology", K.G. Razumovsky Moscow State University of technologies and management (the First Cossack University), Russia.

Morozov Sergey Mikhaylovich, Candidate of Technical Sciences, Associate Professor, Head of the chair "Science and Technical Disciplines and Information Technology", K.G. Razumovsky Moscow State University of technologies and management (the First Cossack University), Russia.

Rogozhina Anna Vladimirovna, Teacher of the chair "Metal and Wooden Structures", National Research Moscow State University of Civil Engineering. Russia.

Keywords: charged particle; non-uniform electric field; point source; mathematical model; differential equations system; point source field.

This article analyzes the behavior of a charged particle in the inhomogeneous electric field of a point source. A mathematical model is constructed and the solution of a system of partial differential equations is given.

удк 331.644

\section{ОБЕСПЕЧЕНИЕ БЕЗОПАСНОСТИ И НАДЕЖНОСТИ ЭЛЕКТРОСНАБЖЕНИЯ ИНЖЕНЕРНО-ТЕХНИЧЕСКИМИ МЕРОПРИЯТИЯМИ}

\begin{abstract}
ОРЛОВ Павел Сергеевич, Ярославская государственная сельскохозяйственная академия ШКРАБАК Владимир Степанович, Санкт-Петербургский государственный аграрный университет
\end{abstract}

БОБКОВ Степан Александрович, Ярославская государственная сельскохозяйственная академия

ШКРАБАК Роман Владимирович, Санкт-Петербургский государственный аграрный университет

ШЕВХУЖЕВ Анатолий Фоадович, ФГБНУ «Северо-Кавказский федеральный научный аграрный изентр»

РУЗАНОВА Наталья Игоревна, Санкт-Петербургский государственный аграрный университет

В статье излагаются пути обеспечения безопасности и надежности электроснабжения инженерно-техническими мероприятиями. Дан всесторонний анализ распространенных инженерно-технических средств безопасности и организационных мероприятий по надежности электроснабжения, соответствующих в основном требованиям нормативно-технической документации (Правилам устройства электроустановок и правилам технической эксплуатации электроустановок). Дан переченъ и анализ инженерно-технических мероприятий, способствующих рещению проблемы обеспечения электробезопасности и надежности электроснабжения предприятия и учреждений. При этом рассмотрены различные сети, используемые в практике электроснабжения разнообразных предприятий. Обращено внимание на составляющие электроснабжения и электропотребления - сети, трансформаторные подстанции, потребители электроэнергии (производство, быт и др.).

Введение. Безопасность и надежность электрообеспечения систем жизнедеятельности предприятий и учреждений страны приоритетный фактор, решающим образом влияющий на все стороны экономики, оборо- носпособности, быта и др. Поэтому им постоянно уделяется внимание во всех направлениях деятельности, что определяет актуальность проблемы в части обеспечения безопасности и надежности электроснабжения (в данном 